\title{
The research of highway safety technology based on Internet of things Wang Bin ${ }^{a}$, Li Jing ${ }^{b *}$, Zhao HuaQi ${ }^{c *}$, Zhang ShiJie ${ }^{d}$
}

\author{
College of Information Science \& Electronic Technology, jiamusi university, Jiamusi,china \\ ajmsuwang@163.com, 'blijing2483@163.com, czhaohuaqi@126.com, ${ }^{d j m s u z s j @ 163 . c o m ~}$
}

Keywords: Internet of things; automobile data recorder; feature extraction

\begin{abstract}
In the design process of automobile data recorder detection system in the intelligent traffic management system, the traditional design method is difficult to resist the interference due to external environment, making the image of automobile data recorder is obscure, resulting in low detection precision problem. Therefore, this paper proposes a design method of automobile data recorder detection system in intelligent traffic management system based on the Internet of things. High precision automobile data recorder is used to make real-time differential dynamic processing for the collected vehicle information. The equivalent spherical correction algorithm is used to make real-time detection on the obtained vehicle image, to get the difference image and then make the binarization processing, and the edge information of tested vehicle can be obtained by statistical method, to realize the automatic recognition of vehicle, ensure the accuracy of detection. Simulation results show that the design method of automobile data recorder detection system in the intelligent traffic management system based on the Internet of things can improve the efficiency of detection, reduce the traffic accidents.
\end{abstract}

\section{Introduction}

At present, the creation of intelligent transportation system of our country is still in the initial stage, because of frequent traffic accidents, it serious impacts on traffic safety of the vehicle [1]. So, deeply intelligent technology, the use of the Internet of things technology, the design of automobile data recorder detection system and reducing or eliminating the interference to the traffic management due to driver's error driving behavior, have become a hot issue of research in the field of transportation, received a lot of attention of experts and scholars [2].

At this stage, in the main intelligent traffic management system, the design methods of vehicle automobile data recorder detection system include the methods based on decision tree algorithm, fuzzy algorithm and neural network algorithm [3-5]. Among them, the most commonly used is the design method of automobile data recorder detection system in intelligent traffic management system based on the distributed algorithm. The design method of automobile data recorder detection system in intelligent traffic management system has broad space for development, and therefore it is paid more attention by a lot of experts and scholars [6].

\section{The Design Method of Automobile Data Recorder Detection System in Intelligent Traffic Management System Based on the Internet of Things}

In the intelligent traffic management system based on the Internet of things, through the camera, the vehicle overlooking real-time image can be obtained by automobile data recorder system, and is transmitted to the sampling monitoring terminal in intelligent traffic management system via message forwarding server. Sampling monitoring terminal uses image processing and recognition technology, to realize the vehicle recognition, generating the random sampling image back to the message server, and transmitted to the sampling machine control system. According to the results, the data stored by real-time monitoring software can be made calculation, judgment, display, query and analysis processing using the analysis processing software. The specific steps are detailed:

(1) The real-time overlooking image of vehicle is obtained by sampling monitoring terminal equipment, and using the equivalent spherical correction algorithm obtain accurate real-time image. The corresponding relationship of image pixel coordinates before and after distortion correction is 
as following:

$$
\begin{aligned}
& x=w / 2+\frac{\left(x^{\prime}-w / z\right)^{2} h \sin \beta}{\sqrt{\left(x^{\prime}-w / z\right)^{2}+\left(y^{\prime}-H 2\right)^{2} \gamma}} \\
& y=H / 2+\frac{Y^{2}\left(Y^{\prime}-H / z\right)^{2} h \sin \beta}{\sqrt{\left(x^{\prime}-w / z\right)^{2}+\left(y^{\prime}-H 2\right)^{2} \gamma}}
\end{aligned}
$$

In the above mentioned formula, $x, y$ and $x^{\prime}, y^{\prime}$ are the corresponding pixel coordinates of the original image and the corrected images respectively. ${ }^{w}, H$ are the width and height pixels in running vehicle image. $\beta$ is the distortion factor, obtained through the tests. $\gamma$ is magnification times, by adjusting this parameter so that the size of acquisition area of vehicle image is moderate. $h$ is the distance from the image plane to the center of lens imaging unit.

(2) Two adjacent frame of images acquired by automobile data recorder in a certain time interval are made difference operation to get difference image.

Differential method is a method to capture the motion of the vehicle contour based on the difference operation of two adjacent frame of images in the video image sequence of automobile data recorder. After obtaining the real-time and accurate overlooking the image of moving vehicle by automobile data recorder, according to internal timer set at $0.05 \mathrm{~S}$, detection system captures the image timely from the automobile data recorder video, and then difference operation is made for the two adjacent piece of mages of vehicle, to obtain the difference picture.

Setting the current acquisition image of automobile data recorder is $B(i, j)$, the background image is $F(i, j)$, the extracted difference image is $T(i, j)$, the formula to obtain difference image can be expressed as:

$$
T(i, j)=\left\{\begin{array}{cc}
F(i, j) & |F(i, j)-B(i, j)| \geq T h \\
255 & |F(i, j)-B(i, j)| \leq T h
\end{array}\right.
$$

In the formula, $T h$ is the judgment threshold, if the absolute difference between the current acquisition images of automobile data recorder $F(i, j)$ and background of $F(i, j)$ is greater than the threshold value $T h$, it is the differential image, otherwise it is non-differential image.

In order to be able to timely capture moving vehicles, it needs to shorten the time of timer, and continue to screenshot pictures to get difference, through continuous cycling pictures capture, the difference images are calculated, through the analysis and judgment of automobile data recorder image information and the time correction for timer, finally the difference image of the two pieces of capture images before and after vehicle stopping can be obtained.

(3) The gray value of the two directions of the treated difference image's rows and columns are made statistical analysis, and the result is judged based on the car quantity.

The above treatment difference images are made edge detection and location, which can get size and coordinate of the vehicle, and determine the location and size of the vehicle, the method is as follows:

The gray threshold $T$ of gray difference image is calculated. Assuming that the width of gray difference image is imgW pixels, height is imgH pixels, then the gray value of $T$ can be calculated by using the following formula:

$$
T=\frac{M+\sigma}{1.1 \sim 1.2}
$$

The following formula is used to one by one judge whether the gray value of each pixel is greater than the T, the pixels which are greater than $\mathrm{T}$ are set to the pure white (255), and the pixels which are less than $\mathrm{T}$ are arranged into a pure black (0), thus obtaining two-value difference image. 


$$
Q_{X Y}=\left\{\begin{array}{cc}
255 & P_{X Y} T \\
0 & P_{X Y} \geq T
\end{array}\right.
$$

After binaryzation of gray difference figure, the moving part in the picture is turned into white, then the white areas are analyzed, to extract the position information of vehicle.

(4) By analyzing, vehicle's boundary and the coordinate's position of image pixel are obtained. Then using image - geodetic coordinate correspondence, the actual position and size of vehicles are obtained, to realize vehicle recognition. The following formula with expression:

$$
\operatorname{right}(j)=\sum_{x-j}^{j-w+1} \operatorname{sgn} 1\left(\operatorname{col}(x)-c_{\max }\right)
$$

Among them, $j$ represents a variable, $j=\operatorname{chaW}-W$.

In the process of vehicle continuous sampling, the vehicle size data obtained in each test is deposited into vehicle information database of sampling system. After every time vehicle identification, the obtained new data and the recent five groups of vehicle detection data in database are made comparative analysis, to verify this vehicle recognition result, and judge the vehicle in the detection image of automobile data recorder whether there is abnormal.

In conclusion, it can be explained, the accuracy and practicability of the design method for automobile data recorder detection system based on the IOT in the intelligent traffic management system are high.

\section{Automatic Recognition of Vehicle Position}

In the situation of the shooting angle of automobile data recorder is fixed, the running region of automobile is relatively fixed in the obtained video screen. In the picture, using a red box circle the relatively fixed running area, and identify whether there are vehicles and vehicle position in the region, the region is known as the vehicle identification area. When designated vehicle identification area, be careful not to let the identification area appear several complete vehicles at the same time.

The basic idea is: in the vehicle recognition area, the column projection of two-value difference image is calculated, using longitudinal characteristics plot matching method, identify the column starting point ${ }^{X_{\text {begin }}}$ and column end point $X_{\text {end }}$ of vehicle location, the vehicle is in the scope of the narrow-band between the $x=x_{\text {begin }}$ and $x=x_{\text {bes }}$ two lines; in the narrow-band range, using the transverse characteristics plot matching method, accurately identify the row starting point $y_{\text {begin }}$ and the row end point ${ }^{y_{\text {end }}}$ of vehicle location, vehicle is at the rectangular region encircled by the 4 straight lines $x=x_{\text {bengin }}, X=x_{\text {end }}, y=y_{\text {begin }}, y=y_{\text {end }}$. Assuming the width of vehicle identification area is chaw, height is $c h a H$, the specific steps of the algorithm is as follows:

Step 1: use the formula below to calculate the column projection $\operatorname{col}(x)$ in vehicle identification area, and define the corresponding function:

$$
\begin{aligned}
& \operatorname{col}(x)=\sum_{y=0}^{\text {chah }-1} \operatorname{sgn} 1\left(Q_{x y}-255\right) \\
& \operatorname{sgn} 1(a)= \begin{cases}1 & \text { if } a, 0 \\
0 & \text { if } a \leq 0\end{cases}
\end{aligned}
$$

Step 2: find out the maximum value ${ }^{C_{\max }}$ in the column projection $\operatorname{col}(x)$ projection, if $C_{\max } \leq \lambda$, it can determine that no car in the vehicle identification area of the image, otherwise, continue to carry out step 3 . Among them, $\lambda$ is the threshold of column projection, its value can be 0.8 times than the length of "small car" in vehicle identification area (unit: pixel). In video footage taken by automobile data recorder, there are 3 kinds of automobile, large-size car (such as buses and trucks), mid-size cars (such as midbus, minibus) and small-size cars (such as car). 
Step 3: followed by calculating the left $(i)$ value of each longitudinal characteristics area in the vehicle identification area, and when calculates a left $(i)$, judging whether it meets the conditions of $\operatorname{left}(i) \geq w^{t}$, if satisfied, setting variable $i^{\prime}=-1$, and immediately go to step 4, otherwise, to calculate the next $\operatorname{left}(i)$, and continue the judgment; if all left $(i)$ does not meet the conditions of the $\operatorname{left}(i) \geq w^{t}$, set the variable $i^{\prime}=-1$. Among them, $i=0,1,2 \ldots$,chaw $-w ; w$ is the width of longitudinal characteristics plot, its value can be the 0.3 times than the width of the "small car" (pixels) in vehicle identification area; ${ }^{C_{\max }}=c_{\max } \times \varphi, \varphi$ is the correction coefficient of the maximum value of column projection, the value can be $0.7 ; w^{t}$ is the width threshold of longitudinal characteristic plot, the value of $0.8 \times w$.

$$
\text { left }=\sum_{x=i}^{i+w-1} \operatorname{sgn} 1\left(\operatorname{col}(x)-c_{\text {max }}\right)
$$

Step 4: to identify the end point of row ${ }^{y_{\text {end }}}$ for the position of the vehicle in narrow-band range.

Step 5: if the value of $y_{\text {begin }}$ and $y_{\text {end }}$ are -1 , it can be considered that no vehicle in the recognition region of the graph; otherwise, it can be judged that there has vehicle in the recognition region of the graph, and the vehicle is circled by the blue square in a rectangular area in which $\left(x_{\text {begin }}, y_{\text {begin }}\right)$ is the left vertex, $\left(x_{\text {eng }}, y_{\text {begin }}\right)$ is right vertex, $\left(x_{\text {begin }}, y_{\text {end }}\right)$ is the left antapex and $\left(x_{\text {end }}, y_{\text {end }}\right)$ is right antapex.

According to the method described above, it can finish the detection according to the image acquired by automobile data recorder, and get the spatial location of vehicle.

\section{Experiment and Simulation Technology}

In order to demonstrate the effectiveness of the design method proposed in this paper for automobile data recorder detection system based on the IOT in the intelligent traffic management system, it needs an experiment, in this experiment, a Visual C++ 6 development environment based on virtual platform is built. In the virtual environment, the traditional algorithm and the proposed algorithm are applied respectively to establish the design model of automobile data recorder detection system in the intelligent traffic management system. According to the experiment, it can be arranged to get figure 1 :

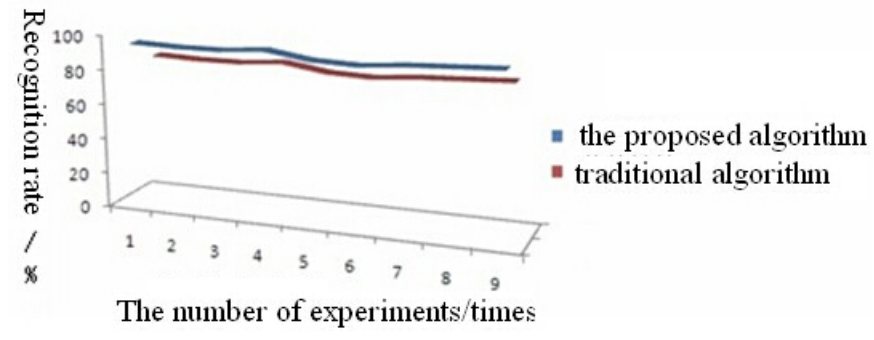

Figure 1 tendency chart of the comparison of recognition rate between different algorithms According to the above figure, it can be arranged to obtain the results in following table: 
Table 1 comparison table of recognition rate between different algorithms

\begin{tabular}{ccc}
\hline $\begin{array}{l}\text { The number of } \\
\text { experiments }\end{array}$ & $\begin{array}{l}\text { Recognition rate of the proposed } \\
\text { algorithm }(\%)\end{array}$ & $\begin{array}{l}\text { Recognition rate of traditional } \\
\text { algorithm }\end{array}$ \\
\hline 1 & 95 & 83 \\
2 & 94 & 82 \\
3 & 94 & 82 \\
4 & 96 & 84 \\
5 & 92 & 80 \\
6 & 91 & 79 \\
7 & 93 & 81 \\
8 & 94 & 82 \\
9 & 95 & 83 \\
\hline
\end{tabular}

Through the above experimental results, it can be showed that the proposed design method of automobile data recorder detection system in intelligent traffic management system based on IOT has high precision.

\section{Conclusion}

Aiming at the problem of which is difficult to resist the external environment interference, making the obscure image of automobile data recorder and low detection precision by using the traditional design method in the design process of automobile data recorder detection system in the intelligent traffic management system, this paper proposes a design method of automobile data recorder detection system in intelligent traffic management system based on the Internet of things. High precision automobile data recorder is used to make real-time differential dynamic processing for the collected vehicle information. The equivalent spherical correction algorithm is used to make real-time detection on the obtained vehicle image, to get the difference image and then make the binarization processing, and the edge information of tested vehicle can be obtained by statistical method, to realize the automatic recognition of vehicle, ensure the accuracy of detection. Simulation results show that the design method of automobile data recorder detection system in the intelligent traffic management system based on the Internet of things can improve the efficiency of detection, reduce the traffic accidents.

\section{Acknowledgement}

This research was supported by the National Nature Foundation of China (Grant No. 51278227), the National Science Foundation of Heilongjiang Province (Grant No. E201212) , the Natural Science Foundation of Heilongjiang Province Education Office(Grant No. 12521554), the National Science Foundation of Jiamusi University(Grant No. Lz2014-005 ) ,the Innovation and Entrepreneurship Training Project for College Students of Heilongjiang Province(Grant No.201410222022) all support is gratefully acknowledged.

\section{References}

[1] Ji Hu, Sun Jixiang, Shao Xiaofang, et al. The Algorithm for Image Edge Detection and Prospect $[\mathrm{J}]$. Computer Engineering and Applications, 2004(14) : 70-73.

[2] Cheng Peiying. A Novel OSTU Segmentation Algorithm for Image Thershold [J] . Computer Applications and Software, 2009( 5) : 227-232.

[3] Yang Qingxiang, Zhang Yong. Determining Orientation of Truck Compartment in Random [J] . Industrial Control Computer, 2007( 8) : 41-42.

[4] Wang Suyu, Shen Lansun. Intelligent Visual Surveillance Technology: A Survey [J]. Journal of Image and Graphics, 2007, 12(9): 1505-1514. 
[5] NickelsK,Hutchinson S. Estimating uncertain thin SSD based featuretracking[J]. Image and Vision Computing, 2002, (20): 47-58.

[6] Zhang Ying, Zhu Jun. Design and Implementation of Automatic Vehicle Identification [J]. Instrument Technique and Sensor, 2006, 1:29-31.

[ 7 ] Ezra, jing-bo wang, Dong Libo. Internet of things in the application of intelligent transportation research [J]. Journal of mobile communications, 2010 (15); 30 - 31.

[8] Yang Libo, liu xiaoming. Chinese and foreign intelligence transportation system development pattern comparison [J]. Journal of comprehensive transportation, 2004 (11) : 64-68.

[9] Huang Suping Jiang Li, qiao, etc. Sensor networks target localization method based on energy [J]. Journal of electroacoustic technology, 2009 (9) : 58-60.

[10] LeiXiaoPing, luo sea. The FPGA in the application of real-time embedded microcomputer data acquisition [J]. Computer application research, 2004 practices (1) : 200-201. 